\title{
Gut Microbiome and the Role of Metabolites in the Study of Graves' Disease
}

\author{
Haihua Liu ${ }^{1,2}$, Huiying Liu ${ }^{1,2}$, Chang Liu ${ }^{1,2}$, Mengxue Shang ${ }^{1,2}$, Tianfu Wei ${ }^{1,2}$ and Peiyuan Yin ${ }^{1 *}$ \\ ${ }^{1}$ Clinical Laboratory of Integrative Medicine, First Affiliated Hospital of Dalian Medical University, Dalian, China, ${ }^{2}$ Institute of \\ Integrative Medicine, Dalian Medical University, Dalian, China
}

Graves' disease (GD) is an autoimmune thyroid disease (AITD), which is one of the most common organ-specific autoimmune disorders with an increasing prevalence worldwide. But the etiology of GD is still unclear. A growing number of studies show correlations between gut microbiota and GD. The dysbiosis of gut microbiota may be the reason for the development of GD by modulating the immune system. Metabolites act as mediators or modulators between gut microbiota and thyroid. The purpose of this review is to summarize the correlations between gut microbiota, microbial metabolites and GD. Challenges in the future study are also discussed. The combination of microbiome and metabolome may provide new insight for the study and put forward the diagnosis, treatment, prevention of GD in the future.

\section{OPEN ACCESS}

Edited by:

Wolfram Weckwerth, University of Vienna, Austria

Reviewed by:

Thomas Kaiser,

University of Minnesota Twin Cities,

United States

${ }^{*}$ Correspondence:

Peiyuan Yin

yinperry@126.com

yimpeiyuan@dm.edu.cn

Specialty section:

This article was submitted to Metabolomics,

a section of the journal

Frontiers in Molecular Biosciences

Received: 22 December 2021

Accepted: 31 January 2022

Published: 16 February 2022

Citation:

Liu H, Liu H, Liu C, Shang M, Wei T and Yin P (2022) Gut Microbiome and the Role of Metabolites in the Study of

Graves' Disease.

Front. Mol. Biosci. 9:841223,

doi: $10.3389 /$ fmolb.2022.841223
Keywords: graves' disease (GD), metabol(n)omics, gut microbiome, autoimmunity, metabolites

\section{INTRODUCTION}

Autoimmune thyroid disease (AITD) are common organ-specific autoimmune disorders with an increasing prevalence worldwide, which involves Hashimoto thyroiditis (HT) and GD (Moshkelgosha et al., 2021). GD is caused by the autoantibodies of the thyrotropin receptor (TSHR), which leads to thyroid hyperplasia and hyperthyroidism (Bahn, 2003; Ishaq et al., 2018; Shi et al., 2019a; Moshkelgosha et al., 2021). Hyperthyroidism, fatigue, weight loss, tachycardia, and heat intolerance are common symptoms of GD. Approximately 50\% of patients may develop Graves' ophthalmopathy (GO), leading to eyelid retractions and exophthalmos (Byeon et al., 2018; Yan et al., 2020 ). GD is the most common cause of $60-80 \%$ of hyperthyroidism and influence about $0.5 \%$ of the general population (Cooper and Stroehla, 2003; Smith and Hegedüs, 2016; Ejtahed et al., 2020). It frequently occurs in the population between 30 and 50 years old. Resemble in other autoimmune diseases, the incidence of GD is higher in women than men, the ratio of about 5/1 (Cooper and Stroehla, 2003; Ji et al., 20188; Nyström et al., 2013; Menconi et al., 2014). The risk factors of GD include genetic predisposition, environmental factors, immune factors (Covelli and Ludgate, 2017).

Hyperthyroidism is a common disease that is difficult to cure completely. Although modern medicine has brought great changes to the prevention, diagnosis, and treatment of autoimmune diseases, the etiology and pathogenesis of these diseases have not been fully illuminated. Abnormal thyroid-related indices often occur repeatedly during clinical treatment (Yang et al., 2019). Furthermore, although current treatment methods for GD can achieve a good effect, clinicians still have some concerns about the choice of treatment for safety reasons (Heyma et al., 1986; Yang et al., 2019). At present, a large number of studies have proved the relationship between intestinal microorganisms and autoimmune diseases, including Type 1 diabetes (Gianchecchi and Fierabracci, 2017; Mullaney et al., 2018), inflammatory bowel disease (Ni et al., 2017; Cao, 2018), systemic lupus 
erythematosus (Corrêa et al., 2017), rheumatoid arthritis (Sato et al., 2017; Teng et al., 2017; Jubair et al., 2018; PicchiantiDiamanti et al., 2018) and autoimmune thyroid disease (Zhou et al., 2014). Metabolites are also considered as important mediators or modulators between gut microbiota and the thyroid. Therefore, metabolomics investigations may provide a new inside view of GD's study.

In this review, we explore the inside relationships between gut microbiota, microbiota-related metabolites and GD, and propose new ideas for prevention, diagnosis, and treatment of GD.

\section{Brife Knowledge of Gut Microbiota}

The human body is a superorganism due to the residence of trillions of prokaryotes symbiosis. Approximately $66 \%$ of the total bacteria are mainly live in the gut. Gut microbiota includes more than one thousand known species of bacteria with at least three million genes (Hehemann et al., 2010; Relman, 2012; Docimo et al., 2020). Apart from absorbing nutrients from the human body that they depend on for survival, intestinal flora also provides beneficial or harmful metabolites to the human body through their metabolic process (Turnbaugh and Gordon, 2009; Relman, 2012). These microflorae participate in the body's energy metabolism through various mechanisms, affecting the conversion of food to energy in the host, and play an essential role in the healthy state of the host (Lozupone et al., 2012; Sommer et al., 2017). When the human body is healthy, microorganisms and various organs and tissues depend on each other and act on either to form a microecological balance and jointly maintain the body's health. If the microecological balance is disturbed, it may lead to disease (Sekirov et al., 2010). Therefore, the intestinal flora is considered an "organ" with multiple regulatory functions, which greatly impacts people's health. Understanding the symbiotic relationship between microorganisms and the human body is of great significance for people to understand their health and the occurrence and development of disease (Turnbaugh and Gordon, 2009; Relman, 2012; Schmidt et al., 2018).

The technological breakthroughs in the microbiome boost the research of gut microbiota. The method of bacterial culture is a restriction of traditional bacterial research. The intestinal flora is cultured with various mediums, and the number of bacterial colonies is measured by dilution and colony count (Lagier et al., 2018). This method is sensitive but is constrained. More than $85 \%$ of the bacteria in the human intestine are anaerobic bacteria, which is difficult to cultivate in the culture medium (Lagier et al., 2015). Recently, the newly established strategy of culturomics enables the culture of microbiota that cannot be cultured before. These new methods initiate the rebirth of culture in microbiology (Kaeberlein et al., 2002; Nichols et al., 2010; Lagier et al., 2018). The development of new techniques has made it possible to study unknown gut flora.16Sr RNA high-throughput sequencing and metagenomics are commonly used methods for detecting gut microbiota. 16Sr RNA sequencing mainly studies the species composition, the evolutionary relationships among species and the diversity of communities (Laudadio et al., 2018). On the basis of $16 \mathrm{Sr}$ RNA sequencing analysis, metagenomic sequencing can also carry out in-depth research on gene and function, and its detection depth can reach the level of species (Wang et al., 2015; Laudadio et al., 2018; Shakya et al., 2019).

With the increasing understanding of the metabolic function of intestinal flora, the narrow sense that host metabolism is regulated by its genes is gradually expanded to co-metabolic regulation of host-symbiotic intestinal bacteria. These metabolites are often from tryptophan metabolic pathways, tyrosine and phenylalanine metabolic pathways, glucose and fatty acid metabolic pathways, classified into indoles, phenols, amino acids, peptides, etc. (Zheng et al., 2011; Van Treuren and Dodd, 2020; Fan and Pedersen, 2021). Microbiome dysbiosis is associated with various diseases, asthma, allergies, inflammatory bowel disease (Arrieta et al., 2015; Bunyavanich et al., 2016; Nishino et al., 2018), autism spectrum disorder (ASD) (Needham et al., 2021), diabetes (Giongo et al., 2011), irritable bowel syndrome (IBS) (Mars et al., 2020), obesity (Schwiertz et al., 2010a), cardiovascular disease (Jie et al., 2017), chronic kidney disease (Sircana et al., 2019). Under different disease states, the species abundance of intestinal flora and its related metabolites have various characteristics. Some studies have found that in patients with IBS, the key findings include an increase in Firmicutes to Bacteroidetes ratio (Krogius-Kurikka et al., 2009; Rajilić-Stojanović et al., 2011; Jeffery et al., 2012l; Mars et al., 2020), a decrease in Bifidobacteria and Lactobacilli (Malinen et al., 2005; Kerckhoffs et al., 2009), and an increase in Ruminococcus and Streptococci species (Kassinen et al., 2007; Rajilić-Stojanović et al., 2011; Saulnier et al., 2011; Hong and Rhee, 2014). A more coincident finding has been decreased alpha diversity. ASD showed lower levels of phylum Firmicutes and a higher abundance of Bacteroidetes (Mangiola et al., 2016; Fattorusso et al., 2019; Sharon et al., 2019). Kang and others observed significant ASD-related behavioral changes in mice with fecal microbiota transplantation (FMT) from ASD (Sharon et al., 2019) and they have developed microbiome transfer therapy (MTT) and observed a reduction in ASD-related symptoms (Kang et al., 2017).

The intestines are also the largest immune organ, gathering more than $70 \%$ of the immune cells as a vital digestive organ. Gut microbiota is also related to the host's immune system (Vatanen et al., 2016). Gut microbiota and metabolites can induce the production of helper T cells (Th) and regulator T cells (Tregs), which contribute to the maturation of host adaptive and innate immunity (Rooks and Garrett, 2016; Shi et al., 2017; Kayama et al., 2020). It can be inferred that autoimmune diseases are closely related to intestinal flora (Levy et al., 2017). There are several studies on the gut microbiota and metabolome among GD patients, and many results strongly support a role for the gut microbiota in GD and GO (Moshkelgosha et al., 2021).

\section{GD and Gut Microbiota}

Some previous studies demonstrated the connections between the gut microbiome and AITD (Köhling et al., 2017). Many studies showed that GD is related to yersinia enterocolitica, e.g., mice fed only with yersinia enterocolitica did not develop GD (Weiss et al., 1983; Wang et al., 2010). There were also significant differences in the microbiota profile between HT patients and healthy controls (Zhao et al., 2018). Zhou et al. characterized the gut microbiota in 
hyperthyroid patients (Zhou et al., 2014). There is limited research on the relationships between Graves' disease and the gut microbiome. However, thyroid hormone levels correlate with the gut microbiome and the diversity of gut bacteria in patients with GD (Ejtahed et al., 2020). Bacteroidetes and Firmicutes are dominant species in the human gut. The ratio of Firmicutes to Bacteroidetes is commonly considered a representative of health status (Chen et al., 2016; Indiani et al., 2018). In the disease state, these two phyla tend to show significant changes. For example, Jiang et al. showed that GD patients had reduced alpha diversity compared with healthy individuals. At the phylum level, GD patients had a significant higher proportion of Bacteroidetes and a significantly lower proportion of Firmicutes than the controls (Jiang et al., 2021). Ishaq et al. also demonstrated this phenomenon in their study (Ishaq et al., 2018). They found that the diversity of gut bacteria in GD patients was less diverse in terms of richness than in healthy people. The proportion of Firmicutes in GD was lower than that in the control group, while the proportion of Bacteroidetes was higher than in the control group (Ishaq et al., 2018). Interestingly, this finding is consistent with what was observed in obese patients. Previous studies have found that obese people tend to have more Firmicutes, while lean people tend to have more Bacteroidetes (Schwiertz et al., 2010b; Riva et al., 2017). Further research work is required about the effects of thyroid hormones on gut microbiota. Besides Firmicutes and Bacteroidetes, there were also significant changes in the ratios and abundances of other phyla. Yan et al. showed that the number of Lactobacillales, Bacilli, Megamonas, Prevotalla and Veillonella strains were increased among GD patients (Yan et al., 2020). However, the number of Rikenellaceae, Ruminococuus and Alistipes strains was decreased among GD patients. In addition, the diversity of gut flora was decreased in patients with GD (Yan et al., 2020). There were also significant changes in gut microbiota in GO patients. Shi et al. found that the bacterial diversity (Simpson and Shannon) was reduced in patients with GO compared to the controls. At the phylum levels, the proportion of Bacteroidetes increased and Firmicutes decreased significantly in GO than that in controls. There were obvious differences in bacterial profiles between the two groups (Shi et al., 2019a). Then, Shi et al. further explored the differences in the compositing of gut microbes between GO and GD patients (Shi et al., 2021). At the phylum levels, the proportion of Chloroflexi was decreased significantly in GO patients. At the genus levels, Bilophila and Subdoligranulum were increased (Shi et al., 2021). It is reported that there are three gut bacteria genera (Bacteroides, Prevotella, Alistipes) that could separate GD patients from healthy individuals with $85 \%$ accuracy (Su et al., 2020).

Thyrotropin receptor antibody (TRAb) is a characteristic indicator of GD, with sensitivity and specificity greater than 95\% for GD diagnosis (Massart et al., 2001; Cooper, 2003). Shi et al. believed that TRAb was significantly correlated with different levels of gut microbiota. At the family level, the proportion of Succinivibrionaceae was positively correlated to TRAb. At the genus level, Subdoligranulum was positively related to TRAb. At the species level, Parabacteroides distasonis showed an opposite correlation with TRAb. Their studies also suggested that GD patients with positive TRAb showed an increased risk of developing GO (Shi et al., 2019a). Prevotella and Bacteroides are positively correlated with TRAb in GO patients (Shi et al., 2019b).

\section{Metabolomics in the Study of GD}

The dynamic balance of Th17 and Treg is closely related to the occurrence and development of various autoimmune diseases (Fasching et al., 2017). Treg cells are a subset of regulatory T cells that regulate the body's autoimmune response. Tregs are characterized by the transcription factor Foxp3 (major regulators of Treg) and mainly exert immune suppressive effects. Maintaining immune homeostasis by secreting inhibitory factors (TGF-B, IL-10, IL-35) mediate immune suppressive effects by regulating TCR signaling promotes secretion and differentiation of anti-inflammatory cytokines (Göschl et al., 2019). The decrease of Treg cells increases the incidence and severity of AITD. And the number of Treg cells is significantly reduced in patients with GD (Saitoh and Nagayama, 2006; Nakano et al., 2007). The Th17 cells are also a subset of T helper cells by secreting interleukin 17 (IL-17, IL-22) induces inflammation and spread. IL-17 is involved in many inflammatory and autoimmune diseases, including systemic and organ-specific autoimmune diseases (Takeuchi et al., 2020; Yasuda et al., 2019). Th17 and IL-17 were increased in GD and participated in the development of GD. In patients with AITD, the proportion of Th17 cells in peripheral blood mononuclear cells (PBMCs) increased and higher mRNA level of their specific transcription factor ROR $\gamma \mathrm{t}$ in PBMCs (Li et al., 2016; Li et al., 2019). However, the level of Tregs and expression of Foxp 3 mRNA were greatly decreased in AITD (Li et al., 2016; Li et al., 2019). Figueroa Vega et al. found that IL-17 was elevated in the thyroid tissues of GD ROR $\gamma t$ mRNA patients, and both IL-17 and IL-22 levels were higher than healthy controls (Figueroa-Vega et al., 2010). Di. Peng observed that the concentration of IL-17 and IL-22 in plasma of GD patients was significantly higher than that of healthy controls, which was consistent with the increase of Th17 cells and positively correlated with TSAb (Peng et al., 2013). However, some studies have shown the opposite results (Yuan et al., 2017). The metabolites of the gut microbiome have been associated with the generation of proinflammatory cytokines and the production of Th17 cells. Commensal bacteria and their metabolites can also promote Treg generation and suppress the immune system (Haase et al., 2018). SCFAs are produced by the fermentation of non-digestible carbohydrates such as dietary fiber by gut bacteria, including butyrate (C (Shi et al., 2019a)), propionate (C (Ishaq et al., 2018)) and acetate (C (Bahn, 2003)), are essential metabolites in maintaining homeostasis (Luu and Visekruna, 2019). SCFAs have been proved to alter chemotaxis and phagocytosis, changes in cell function and proliferation, induction of reactive oxygen species (ROS), anti-tumor and anti-inflammatory (Tan et al., 2014). SCFAs contribute to the maintenance of intestinal barrier integrity and its regeneration effect on the intestinal epithelium (Memba et al., 2017). SCFAs are valuable sources of nutrients for enterocytes, together with thyroid hormones (chiefly triiodothyronine), stimulating 


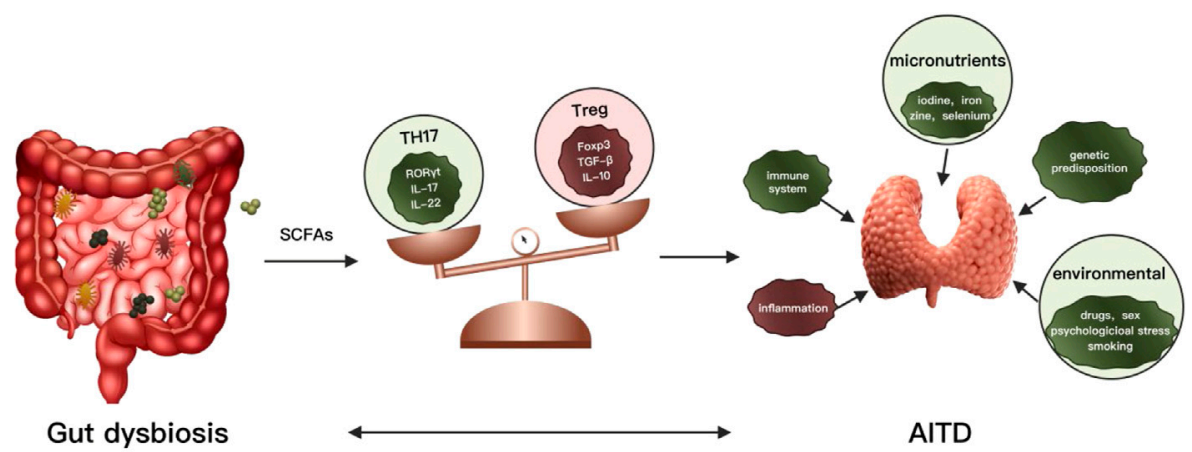

FIGURE 1 | Association between gut microbiota, metabolites, and thyroid autoimmune diseases.

enterocyte differentiation (Cayres et al., 2021; Meng et al., 1999). It also increases intercellular integrity and reduces the risk of a "leaky gut" by improving the adhesion of intestinal cells and reducing the $\mathrm{PH}$ in the intestinal tract, thus avoiding the invasion of pathological organisms (Memba et al., 2017; Bargiel et al., 2021). It is suggested that GD's development is often linked to a compromised intestinal barrier (Knezevic et al., 2020). Recent studies emphasized the immunomodulatory potential of SCFAs in various autoimmune diseases and inflammatory disorders such as multiple sclerosis (MS), colitis, rheumatoid arthritis and AITD. The relation between SCFAs and thyroid function seems to be confirmed by several studies in the scientific literature describing changes in the gut microbiota, including concentrations of SCFAs in impaired thyroid status (Virili et al., 2018; Liu et al., 2020). Currently, two essential functions for SCFAs have been identified, inhibition of histone deacetylases (HDACs) and activation of G-protein coupled receptors (GPCRs), particularly GPR43, GPR41 and GPR109 A (Tan et al., 2014) (Sivaprakasam et al., 2016). Butyrate has been shown to have a positive effect on rheumatoid arthritis (Hui et al., 2019), inflammatory bowel disease (IBD) (Zhou et al., 2018) and autoimmune hepatitis (AIH) (Hu et al., 2018) by rebalancing between Treg and Th17 and increasing the number of Treg cells and decreasing Th17 cells in the system (Figure 1). Propionate is found to affect multiple sclerosis (MS) (Duscha et al., 2020) and GD (Su et al., 2020). However, little is known about the role of the SCFAs in Graves' disease.

Struja et al. predicted the relapse of hyperthyroidism based on the assessment of metabonomics differences. Pyruvate and triglycerides are considered as predictors with AUCs of 0.73 and 0.67 (Struja et al., 2018). Al-Majdoub and others reported changes in the carnitine metabolism of GD patients prior to treatment compared to posttreatment (Al-Majdoub et al., 2017). The level of short-chain acylcarnitine decreased, medium-chain acylcarnitine increased, and long-chain acylcarnitine remained unchanged. The authors speculated that these phenomena reflect a starvation process that induced by hyperthyroidism (AlMajdoub et al., 2017). Lipid profile from plasma and urine samples of GD patients was significantly different compared to controls. Some of Glycerophosphoethanolamine (PE), Glycerophosphoinositol (PI), Triacylglycerol (TG) and
Glycerophosphoglycerol (PG) have changed significantly (Byeon et al., 2018). Polyamine metabolic profiles are also altered in AITD. GD and HT patients showed the same change relative to the control group for most of the polyamine metabolites. L-arginine (L-ARG), L-omithine (L-ORN), lysine (LYS) agmatine (AGM) are significantly and $\mathrm{N}$-acetylputrescine (NPUT), spermine (SPM), 1,3diaminopropane (DAP) are lower than the control group. However, GD and HT have different characteristics of change. GD patients had significantly lower cadverine (CAD) and higher $\mathrm{N}$-acetylspermidine (NSPD), spermidine (SPD) and r-Aminobutyric (GABA) acid than the control group. But $\mathrm{N}$-acetylspermine (NSPM) was decreased in HT. The antiinflammatory effect of SPM was better than that of SDP. SPM/SPD can be more effective for estimating the antiinflammatory effect. A decrease in SPM/SPD in patients with AITD indicated reduce in protective polyamines. SPM/SPD was negatively correlated with inflammatory chemokine IP-10 and TPOAb (Rider et al., 2007; Song et al., 2019). Ji et al. performed a non-targeted metabolomics analysis on the blood and orbital tissues of GD, GO and healthy controls. They identified ten differential metabolites in the disease group (gluconic acid, glucose, pelargonic acid, threose, fumaric acid, glycerol, mannose, pentade canoic acid, pyruvate, and 2- (4hydroxyphenyl)ethanol) (Ji et al., 20188). The metabolite panel achieved an accuracy of 0.931 and the sensitivity and specificity are 0.787 and 0.875 , respectively (Ji et al., 20188). Among the metabolite panel, almost all metabolites showed a positive correlation with the levels of TRAb (Ji et al., 20188). Propionate was significantly reduced in GD patients, which was negatively correlated with FT3, FT4, TRAb level, and positively correlated with TSH level (Su et al., 2020). At present, there are not many studies on GD metabolomics, and the specific association and mechanism still need to be further studied.

Gut dysbiosis can lead to changes in metabolites such as SCFAs. As a consequence, the balance of Th17 and Tregs would be damaged, leading to an autoimmune response and causing autoimmune thyroid diseases. AITD: autoimmune thyroid diseases; IL: interleukin; Th: T helper cell; Tregs: regulatory $\mathrm{T}$ cells. 


\section{Microbiome and Metabolome in GD Study}

In the last 20 years, it has been established that the gut microbiome plays an essential role in maintaining host health and the occurrence and progression of the disease. Metabolites are the primary way that gut microbes interact with hosts. The small molecules generated or modified from microorganisms can be detected in urine, serum, feces, cerebrospinal fluid, and other tissues (Holmes et al., 2011; Del Rio et al., 2017). The homeostasis of a healthy intestinal environment is regulated by the balance of microbiota, metabolites, and immune systems. In the state of disease, the intestinal balance is usually destroyed. Studies showed that gut dysbiosis leads to Treg/Th17 imbalance through the propionic acid regulation pathway, which, together with other pathogenic factors, promotes GD occurrence (Su et al., 2020). Gut dysbiosis was mainly manifested by a significant decrease in SCFAs-producing bacteria and SCFAs. Bacteroides fragilis YCH46 strain in GD patients was obviously reduced compared to healthy controls. It can produce propionic acid, increase the number of Treg cells and reduce the number of Th17 cells. Therefore, $B$. fragilis YCH46 was a natural activator of Treg cells and inhibitor of Th17 cells (Rios-Covian et al., 2015). YCH46 strain of $B$. fragilis provides a new direction for the treatment of GD. It can improve immune dysfunction in GD patients and be used as an immunomodulator or as an auxiliary treatment for GD patients to reduce recurrence rate (Su et al., 2020). A recent study found significant differences in metabolic pathways between GD groups and healthy controls. Formaldehyde assimilation and allantoin degradation, mevalonate and isoprene biosynthesis significantly increased in the GD patients. In contrast, the microbial metabolic abilities of fatty acid biosynthesis, pyruvate fermentation to hexanol, anaerobic energy metabolism, creatinine degradation and gluconeogenesis decreased significantly in relative abundance in the patients. The change of gut microbiota is Butyricimonas faecalis, Faecalibacterium prausnitzii, Akkermansia muciniphila and Bifidobacterium adolescentis decreased in the GD, whereas Veillonella parvula, Eggerthella lenta, Fusobacterium mortiferum, Streptococcus parasanguinis, and Streptococcus salivarius were enriched. And use propionic acid, acetic acid, cholate and chenodeoxycholate as potential biomarkers (Zhu et al., 2021). Jiang et al. found that Blautia, Eubacterium and Anaerostipes were decreased in GD. Eubacterium and Anaerostipes produce butyric acid and maintain the integrity of the intestinal epithelium as well as induce the generate of Treg

\section{REFERENCES}

Al-Majdoub, M., Lantz, M., and Spégel, P. (2017). Treatment of Swedish Patients with Graves' Hyperthyroidism Is Associated with Changes in Acylcarnitine Levels. Thyroid 27, 1109-1117. doi:10.1089/thy.2017.0218

Arrieta, M.-C., Stiemsma, L. T., Dimitriu, P. A., Thorson, L., Russell, S., YuristDoutsch, S., et al. (2015). Early Infancy Microbial and Metabolic Alterations Affect Risk of Childhood Asthma. Sci. Transl. Med. 7, 307ra152. doi:10.1126/ scitranslmed.aab2271

Bahn, R. S. (2003). Pathophysiology of Graves' Ophthalmopathy: The Cycle of Disease. J. Clin. Endocrinol. Metab. 88, 1000-1946. doi:10.1210/jc.2002-030010 cells to strengthen the tightness of the intestinal mucosal barrier (Duncan et al., 2004; Venkataraman et al., 2016; Jiang et al., 2021). The primary metabolite of Blautia is butyric acid and has been shown to have anti-inflammatory effects (Jenq et al., 2015). The decrease of these three butyric acid-producing bacteria leads to the reduction of butyric acid and inhibits the differentiation of Treg cells, resulting in immune system dysfunction and eventually the development of AITD (Jiang et al., 2021).

\section{DISCUSSION}

Autoimmune diseases are still challenging for the clinic. Changes in the composition and abundance of the gut microbiota, as well as related metabolites, are closely linked to the occurrence of GD. These findings provide some potential biomarkers for early diagnosis of GD, and some new probiotics related to GD can be used for adjunctive treatment and prevention of recurrence. However, related studies on gut microbiota metabolome in patients with GD are relatively lacking, and further studies are needed. It is believed that probiotics have positive effects on thyroid diseases, which has been confirmed in vitro cell studies and animal studies. However, these effects on human beings still require intensive investigations. Accurate qualitativequantitative characterization of probiotics according to different pathological stages are also needed. Current metabolomics studies provide the correlations between gut micrbiota and the disease, however, the molecular mechanism between gut microbiota and GD remain unclear. One of the key point is how the metabolites synthesized by the gut microbiota. This is essential for the following development of related medicines.

The ultimate goal for the multi-omics study is to develop new diagnostic standards (microbial/metabolite biomarkers) and treatment strategies (probiotics/targeted microbial therapy or functional metabolites) for GD, with an individual treatment plan for each patient to achieve a complete cure and prevent a recurrence.

\section{AUTHOR CONTRIBUTIONS}

HaL write the manuscript, PY revised the paper. HuL, CL, MS, and TW review the manuscript.

Bargiel, P., Szczuko, M., Stachowska, L., Prowans, P., Czapla, N., Markowska, M., et al. (2021). Microbiome Metabolites and Thyroid Dysfunction. Jcm 10, 3609. doi:10.3390/jcm10163609

Bunyavanich, S., Shen, N., Grishin, A., Wood, R., Burks, W., Dawson, P., et al. (2016). Early-life Gut Microbiome Composition and Milk Allergy Resolution. J. Allergy Clin. Immunol. 138, 1122-1130. doi:10.1016/j.jaci.2016.03.041

Byeon, S. K., Park, S. H., Lee, J. C., Hwang, S., Ku, C. R., Shin, D. Y., et al. (2018). Lipidomic Differentiation of Graves' Ophthalmopathy in Plasma and Urine from Graves' Disease Patients. Anal. Bioanal. Chem. 410, 7121-7133. doi:10. 1007/s00216-018-1313-2

Cao, S. S. (2018). Cellular Stress Responses and Gut Microbiota in Inflammatory Bowel Disease. Gastroenterol. Res. Pract. 2018, 1-13. doi:10.1155/2018/7192646 
Cayres, L. C. d. F., de Salis, L. V. V., Rodrigues, G. S. P., Lengert, A. v. H., Biondi, A. P. C., Sargentini, L. D. B., et al. (2021). Detection of Alterations in the Gut Microbiota and Intestinal Permeability in Patients with Hashimoto Thyroiditis. Front. Immunol. 12, 579140. doi:10.3389/fimmu.2021.579140

Chen, S., Cheng, H., Wyckoff, K. N., and He, Q. (2016). Linkages of Firmicutes and Bacteroidetes Populations to Methanogenic Process Performance. J. Ind. Microbiol. Biotechnol. 43, 771-781. doi:10.1007/s10295-016-1760-8

Cooper, D. S. (2003). Hyperthyroidism. The Lancet 362, 459-468. doi:10.1016/ s0140-6736(03)14073-1

Cooper, G. S., and Stroehla, B. C. (2003). The Epidemiology of Autoimmune Diseases. Autoimmun. Rev. 2 (3), 119-125. doi:10.1016/s1568-9972(03)00006-5

Corrêa, J. D., Calderaro, D. C., Ferreira, G. A., Mendonça, S. M. S., Fernandes, G. R., Xiao, E., et al. (2017). Subgingival Microbiota Dysbiosis in Systemic Lupus Erythematosus: Association with Periodontal Status. Microbiome 5, 34. doi:10. 1186/s40168-017-0252-Z

Covelli, D., and Ludgate, M. (2017). The Thyroid, the Eyes and the Gut: a Possible Connection. J. Endocrinol. Invest. 40, 567-576. doi:10.1007/s40618-016-0594-6

Del Rio, D., Zimetti, F., Caffarra, P., Tassotti, M., Bernini, F., Brighenti, F., et al. (2017). The Gut Microbial Metabolite Trimethylamine-N-Oxide Is Present in Human Cerebrospinal Fluid. Nutrients 9, 1053. doi:10.3390/nu9101053

Docimo, G., Cangiano, A., Romano, R. M., Pignatelli, M. F., Offi, C., Paglionico, V. A., et al. (2020). The Human Microbiota in Endocrinology: Implications for Pathophysiology, Treatment, and Prognosis in Thyroid Diseases. Front. Endocrinol. 11, 586529. doi:10.3389/fendo.2020.586529

Duncan, S. H., Louis, P., and Flint, H. J. (2004). Lactate-utilizing Bacteria, Isolated from Human Feces, that Produce Butyrate as a Major Fermentation Product. Appl. Environ. Microbiol. 70 (10), 5810-5817. doi:10.1128/AEM.70.10.58105817.2004

Duscha, A., Gisevius, B., Hirschberg, S., Yissachar, N., Stangl, G. I., Eilers, E., et al. (2020). Propionic Acid Shapes the Multiple Sclerosis Disease Course by an Immunomodulatory Mechanism. Cell 180, 1067-1080. e16. doi:10.1016/j.cell. 2020.02.035

Ejtahed, H.-S., Angoorani, P., Soroush, A.-R., Siadat, S.-D., Shirzad, N., HasaniRanjbar, S., et al. (2020). Our Little Friends with Big Roles: Alterations of the Gut Microbiota in Thyroid Disorders. Emiddt 20, 344-350. doi:10.2174/ 1871530319666190930110605

Fan, Y., and Pedersen, O. (2021). Gut Microbiota in Human Metabolic Health and Disease. Nat. Rev. Microbiol. 19, 55-71. doi:10.1038/s41579-020-0433-9

Fasching, P., Stradner, M., Graninger, W., Dejaco, C., and Fessler, J. (2017). Therapeutic Potential of Targeting the Th17/Treg Axis in Autoimmune Disorders. Molecules 22, 134. doi:10.3390/molecules22010134

Fattorusso, A., Di Genova, L., Dell'Isola, G., Mencaroni, E., and Esposito, S. (2019). Autism Spectrum Disorders and the Gut Microbiota. Nutrients 11, 521. doi:10. 3390/nu11030521

Figueroa-Vega, N., Alfonso-Pe'rez, M., Benedicto, I., Sa'nchez-Madrid, F., Gonza' lez-Amaro, R., and Marazuela, M. (2010). Increased Circulating Proinflammatory Cytokines and Th17 Lymphocytes in Hashimoto's Thyroiditis. J. Clin. Endocrinol. Metab. 95, 953-962. doi:10.1210/jc.2009-1719

Gianchecchi, E., and Fierabracci, A. (2017). On the Pathogenesis of Insulindependent Diabetes Mellitus: the Role of Microbiota. Immunol. Res. 65 (1), 242-256. doi:10.1007/s12026-016-8832-8

Giongo, A., Gano, K. A., Crabb, D. B., Mukherjee, N., Novelo, L. L., Casella, G., et al. (2011). Toward Defining the Autoimmune Microbiome for Type 1 Diabetes. ISME J. 5, 82-91. doi:10.1038/ismej.2010.92

Göschl, L., Scheinecker, C., and Bonelli, M. (2019). Treg Cells in Autoimmunity: from Identification to Treg-Based Therapies. Semin. Immunopathol 41, 301-314. doi:10.1007/s00281-019-00741-8

Haase, S., Haghikia, A., Wilck, N., Müller, D. N., and Linker, R. A. (2018). Impacts of Microbiome Metabolites on Immune Regulation and Autoimmunity. Immunology 154, 230-238. doi:10.1111/imm.12933

Hehemann, J.-H., Correc, G., Barbeyron, T., Helbert, W., Czjzek, M., and Michel, G. (2010). Transfer of Carbohydrate-Active Enzymes from marine Bacteria to Japanese Gut Microbiota. Nature 464, 908-912. doi:10.1038/nature08937

Heyma, P., Harrison, L. C., and Robins-Browne, R. (1986). Thyrotrophin (TSH) Binding Sites on Yersinia Enterocolitica Recognized by Immunoglobulins from Humans with Graves' Disease. Clin. Exp. Immunol. 64 (2), 249-254.

Holmes, E., Li, J. V., Athanasiou, T., Ashrafian, H., and Nicholson, J. K. (2011). Understanding the Role of Gut Microbiome-Host Metabolic Signal Disruption in Health and Disease. Trends Microbiol. 19, 349-359. doi:10.1016/j.tim.2011. 05.006

Hong, S. N., and Rhee, P-L. (2014). Unraveling the Ties between Irritable Bowel Syndrome and Intestinal Microbiota. Wjg 20, 2470-2481. doi:10.3748/wjg.v20. i10.2470

Hu, E.-D., Chen, D.-Z., Wu, J.-L., Lu, F.-B., Chen, L., Zheng, M.-H., et al. (2018). High Fiber Dietary and Sodium Butyrate Attenuate Experimental Autoimmune Hepatitis through Regulation of Immune Regulatory Cells and Intestinal Barrier. Cell Immunol. 328, 24-32. doi:10.1016/j.cellimm.2018.03.003

Hui, W., Yu, D., Cao, Z., and Zhao, X. (2019). Butyrate Inhibit Collagen-Induced Arthritis via Treg/IL-10/Th17 axis. Int. Immunopharmacology 68, 226-233. doi:10.1016/j.intimp.2019.01.018

Indiani, C. M. d. S. P., Rizzardi, K. F., Castelo, P. M., Ferraz, L. F. C., Darrieux, M., and Parisotto, T. M. (2018). Childhood Obesity and Firmicutes/Bacteroidetes Ratio in the Gut Microbiota: A Systematic Review. Child. Obes. 14 (8), 501-509. doi:10.1089/chi.2018.0040

Ishaq, H. M., Mohammad, I. S., Shahzad, M., Ma, C., Raza, M. A., Wu, X., et al. (2018). Molecular Alteration Analysis of Human Gut Microbial Composition in Graves' Disease Patients. Int. J. Biol. Sci. 14, 1558-1570. doi:10.7150/ijbs.24151

Jeffery, I. B., O’Toole, P. W., Öhman, L., Claesson, M. J., Deane, J., Quigley, E. M. M., et al. (2012l). An Irritable Bowel Syndrome Subtype Defined by Speciesspecific Alterations in Faecal Microbiota. Gut 61 (7), 997-1006. doi:10.1136/ gutjnl-2011-301501

Jenq, R. R., Taur, Y., Devlin, S. M., Ponce, D. M., Goldberg, J. D., Ahr, K. F., et al. (2015). Intestinal Blautia Is Associated with Reduced Death from Graft-VersusHost Disease. Biol. Blood Marrow Transplant. 21, 1373-1383. doi:10.1016/j. bbmt.2015.04.016

Ji, D. Y., Park, S. H., Park, S. J., Kim, K. H., Ku, C. R., Shin, D. Y., et al. (2018). Comparative Assessment of Graves' Disease and Main Extrathyroidal Manifestation, Graves' Ophthalmopathy, by Non-targeted Metabolite Profiling of Blood and Orbital Tissue. Sci. Rep. 8, 9262. doi:10.1038/s41598018-27600-0

Jiang, W., Yu, X., Kosik, R. O., Song, Y., Qiao, T., Tong, J., et al. (2021). Gut Microbiota May Play a Significant Role in the Pathogenesis of Graves' Disease. Thyroid 31, 810-820. doi:10.1089/thy.2020.0193

Jie, Z., Xia, H., Zhong, S.-L., Feng, Q., Li, S., Liang, S., et al. (2017). The Gut Microbiome in Atherosclerotic Cardiovascular Disease. Nat. Commun. 8, 845 doi:10.1038/s41467-017-00900-1

Jubair, W. K., Hendrickson, J. D., Severs, E. L., Schulz, H. M., Adhikari, S., Ir, D., et al. (2018). Modulation of Inflammatory Arthritis in Mice by Gut Microbiota through Mucosal Inflammation and Autoantibody Generation. Arthritis Rheumatol. 70, 1220-1233. doi:10.1002/art.40490

Kaeberlein, T., Lewis, K., and Epstein, S. S. (2002). Isolating "Uncultivable" Microorganisms in Pure Culture in a Simulated Natural Environment. Science 296, 1127-1129. doi:10.1126/science.1070633

Kang, D.-W., Adams, J. B., Gregory, A. C., Borody, T., Chittick, L., Fasano, A., et al. (2017). Microbiota Transfer Therapy Alters Gut Ecosystem and Improves Gastrointestinal and Autism Symptoms: an Open-Label Study. Microbiome 5, 10. doi:10.1186/s40168-016-0225-7

Kassinen, A., Krogius-Kurikka, L., Mäkivuokko, H., Rinttilä, T., Paulin, L., Corander, J., et al. (2007). The Fecal Microbiota of Irritable Bowel Syndrome Patients Differs Significantly from that of Healthy Subjects. Gastroenterology 133, 24-33. doi:10.1053/j.gastro.2007.04.005

Kayama, H., Okumura, R., and Takeda, K. (2020). Interaction between the Microbiota, Epithelia, and Immune Cells in the Intestine. Annu. Rev. Immunol. 38, 23-48. doi:10.1146/annurev-immunol-070119-115104

Kerckhoffs, A. P., Samsom, M., van der Rest, M. E., de Vogel, J., Knol, J., Ben-Amor, K., et al. (2009). Lower Bifidobacteria Counts in Both Duodenal MucosaAssociated and Fecal Microbiota in Irritable Bowel Syndrome Patients. Wjg 15, 2887. doi:10.3748/wjg.15.2887

Knezevic, J., Starchl, C., Tmava Berisha, A., and Amrein, K. (2020). Thyroid-GutAxis: How Does the Microbiota Influence Thyroid Function? Nutrients 12, 1769. doi:10.3390/nu12061769

Köhling, H. L., Plummer, S. F., Marchesi, J. R., Davidge, K. S., and Ludgate, M. (2017). The Microbiota and Autoimmunity: Their Role in Thyroid Autoimmune Diseases. Clin. Immunol. 183, 63-74. doi:10.1016/j.clim.2017. 07.001 
Krogius-Kurikka, L., Lyra, A., Malinen, E., Aarnikunnas, J., Tuimala, J., Paulin, L., et al. (2009). Microbial Community Analysis Reveals High Level Phylogenetic Alterations in the Overall Gastrointestinal Microbiota of DiarrhoeaPredominant Irritable Bowel Syndrome Sufferers. BMC Gastroenterol. 9, 95. doi:10.1186/1471-230X-9-95

Lagier, J.-C., Dubourg, G., Million, M., Cadoret, F., Bilen, M., Fenollar, F., et al. (2018). Culturing the Human Microbiota and Culturomics. Nat. Rev. Microbiol. 16, 540-550. doi:10.1038/s41579-018-0041-0

Lagier, J.-C., Hugon, P., Khelaifia, S., Fournier, P.-E., La Scola, B., and Raoult, D. (2015). The Rebirth of Culture in Microbiology through the Example of Culturomics to Study Human Gut Microbiota. Clin. Microbiol. Rev. 28, 237-264. doi:10.1128/CMR.00014-14

Laudadio, I., Fulci, V., Palone, F., Stronati, L., Cucchiara, S., and Carissimi, C. (2018). Quantitative Assessment of Shotgun Metagenomics and 16S rDNA Amplicon Sequencing in the Study of Human Gut Microbiome. OMICS: A J. Integr. Biol. 22, 248-254. doi:10.1089/omi.2018.0013

Levy, M., Kolodziejczyk, A. A., Thaiss, C. A., and Elinav, E. (2017). Dysbiosis and the Immune System. Nat. Rev. Immunol. 17, 219-232. doi:10.1038/nri.2017.7

Li, C., Yuan, J., Zhu, Y.-f., Yang, X.-j., Wang, Q., Xu, J., et al. (2016). Imbalance of Th17/Treg in Different Subtypes of Autoimmune Thyroid Diseases. Cell Physiol Biochem 40, 245-252. doi:10.1159/000452541

Li, Q., Wang, B., Mu, K., and Zhang, J. A. (2019). The Pathogenesis of Thyroid Autoimmune Diseases: New T Lymphocytes - Cytokines Circuits beyond the Th1-Th2 Paradigm. J. Cell Physiol 234, 2204-2216. doi:10.1002/jcp.27180

Liu, S., An, Y., Cao, B., Sun, R., Ke, J., and Zhao, D. (2020). The Composition of Gut Microbiota in Patients Bearing Hashimoto's Thyroiditis with Euthyroidism and Hypothyroidism. Int. J. Endocrinol. 2020, 1-9. doi:10.1155/2020/5036959

Lozupone, C. A., Stombaugh, J. I., Gordon, J. I., Jansson, J. K., and Knight, R. (2012). Diversity, Stability and Resilience of the Human Gut Microbiota. Nature 489, 220-230. doi:10.1038/nature11550

Luu, M., and Visekruna, A. (2019). Short-chain Fatty Acids: Bacterial Messengers Modulating the Immunometabolism of T Cells. Eur. J. Immunol. 49, 842-848. doi:10.1002/eji.201848009

Malinen, E., Rinttila, T., Kajander, K., Matto, J., Kassinen, A., Krogius, L., et al. (2005). Analysis of the Fecal Microbiota of Irritable Bowel Syndrome Patients and Healthy Controls with Real-Time PCR. Am. J. Gastroenterol. 100 (2), 373-382. doi:10.1111/j.1572-0241.2005.40312.x

Mangiola, F., Ianiro, G., Franceschi, F., Fagiuoli, S., Gasbarrini, G., and Gasbarrini, A. (2016). Gut Microbiota in Autism and Mood Disorders. Wjg 22, 361-368. doi:10.3748/wjg.v22.i1.361

Mars, R. A. T., Yang, Y., Ward, T., Houtti, M., Priya, S., Lekatz, H. R., et al. (2020). Longitudinal Multi-Omics Reveals Subset-specific Mechanisms Underlying Irritable Bowel Syndrome. Cell 182, 1460-1473. e17. doi:10.1016/j.cell.2020. 08.007

Massart, C., Orgiazzi, J., and Maugendre, D. (2001). Clinical Validity of a New Commercial Method for Detection of TSH-Receptor Binding Antibodies in Sera from Patients with Graves' Disease Treated with Antithyroid Drugs. Clinica Chim. Acta 304, 39-47. doi:10.1016/s0009-8981(00)00385-5

Memba, R., Duggan, S. N., Ni Chonchubhair, H. M., Griffin, O. M., Bashir, Y., O'Connor, D. B., et al. (2017). The Potential Role of Gut Microbiota in Pancreatic Disease: A Systematic Review. Pancreatology 17, 867-874. doi:10. 1016/j.pan.2017.09.002

Menconi, F., Marcocci, C., and Marinò, M. (2014). Diagnosis and Classification of Graves' Disease. Autoimmun. Rev. 13 (4-5), 398-402. doi:10.1016/j.autrev.2014. 01.013

Meng, S., Wu, J. T., Archer, S. Y., and Hodin, R. A. (1999). Short-chain Fatty Acids and Thyroid Hormone Interact in Regulating Enterocyte Gene Transcription. Surgery 126, 293-298. doi:10.1016/s0039-6060(99)70168-6

Moshkelgosha, S., Verhasselt, H. L., Verhasselt, H. L., Masetti, G., Covelli, D., Biscarini, F., et al. (2021). Modulating Gut Microbiota in a Mouse Model of Graves' Orbitopathy and its Impact on Induced Disease. Microbiome 9 (1), 45. doi:10.1186/s40168-020-00952-4

Mullaney, J. A., Stephens, J. E., Costello, M.-E., Fong, C., Geeling, B. E., Gavin, P. G., et al. (2018). Correction to: Type 1 Diabetes Susceptibility Alleles Are Associated with Distinct Alterations in the Gut Microbiota. Microbiome 6, 51. doi:10.1186/s40168-018-0438-z

Nakano, A., Watanabe, M., Iida, T., Kuroda, S., Matsuzuka, F., Miyauchi, A., et al. (2007). Apoptosis-induced Decrease of Intrathyroidal CD4+CD25+ Regulatory
T Cells in Autoimmune Thyroid Diseases. Thyroid 17, 25-31. doi:10.1089/thy. 2006.0231

Needham, B. D., Adame, M. D., Serena, G., Rose, D. R., Preston, G. M., Conrad, M. C., et al. (2021). Plasma and Fecal Metabolite Profiles in Autism Spectrum Disorder. Biol. Psychiatry 89, 451-462. doi:10.1016/j.biopsych.2020.09.025

Ni, J., Wu, G. D., Albenberg, L., and Tomov, V. T. (2017). Gut Microbiota and IBD: Causation or Correlation? Nat. Rev. Gastroenterol. Hepatol. 14, 573-584. doi:10. 1038/nrgastro.2017.88

Nichols, D., Cahoon, N., Trakhtenberg, E. M., Pham, L., Mehta, A., Belanger, A., et al. (2010). Use of Ichip for High-Throughput In Situ Cultivation of "Uncultivable" Microbial Species. Appl. Environ. Microbiol. 76, 2445-2450. doi:10.1128/AEM.01754-09

Nishino, K., Nishida, A., Inoue, R., Kawada, Y., Ohno, M., Sakai, S., et al. (2018). Analysis of Endoscopic brush Samples Identified Mucosa-Associated Dysbiosis in Inflammatory Bowel Disease. J. Gastroenterol. 53, 95-106. doi:10.1007/ s00535-017-1384-4

Nyström, H. F., Jansson, S., and Berg, G. (2013). Incidence Rate and Clinical Features of Hyperthyroidism in a Long-Term Iodine Sufficient Area of Sweden (Gothenburg) 2003-2005. Clin. Endocrinol. 78, 768-776. doi:10.1111/cen.12060

Peng, D., Xu, B., Wang, Y., Guo, H., and Jiang, Y. (2013). A High Frequency of Circulating Th22 and Th17 Cells in Patients with New Onset Graves' Disease. PLoS One 8, e68446. doi:10.1371/journal.pone.0068446

Picchianti-Diamanti, A., Panebianco, C., Salemi, S., Sorgi, M., Di Rosa, R., Tropea, A., et al. (2018). Analysis of Gut Microbiota in Rheumatoid Arthritis Patients: Disease-Related Dysbiosis and Modifications Induced by Etanercept. Ijms 19, 2938. doi:10.3390/ijms19102938

Rajilić-Stojanović, M., Biagi, E., Heilig, H. G., Kajander, K., Kekkonen, R. A., Tims, S., et al. (2011). Global and Deep Molecular Analysis of Microbiota Signatures in Fecal Samples from Patients with Irritable Bowel Syndrome. Gastroenterology 141 (5), 1792-1801. doi:10.1053/j.gastro.2011.07.043

Relman, D. A. (2012). The Human Microbiome: Ecosystem Resilience and Health. Nutr. Rev. 70 (Suppl. 1), S2-S9. doi:10.1111/j.1753-4887.2012.00489.x

Rider, J. E., Hacker, A., Mackintosh, C. A., Pegg, A. E., Woster, P. M., and Casero, R. A. (2007). Spermine and Spermidine Mediate protection against Oxidative Damage Caused by Hydrogen Peroxide. Amino Acids 33, 231-240. doi:10.1007/ s00726-007-0513-4

Rios-Covian, D., Sánchez, B., Salazar, N., Martínez, N., Redruello, B., Gueimonde, M., et al. (2015). Different Metabolic Features of Bacteroides Fragilis Growing in the Presence of Glucose and Exopolysaccharides of Bifidobacteria. Front. Microbiol. 6, 825. doi:10.3389/fmicb.2015.00825

Riva, A., Borgo, F., Lassandro, C., Verduci, E., Morace, G., Borghi, E., et al. (2017). Pediatric Obesity Is Associated with an Altered Gut Microbiota and Discordant Shifts in F Irmicutes Populations. Environ. Microbiol. 19, 95-105. doi:10.1111/ $1462-2920.13463$

Rooks, M. G., and Garrett, W. S. (2016). Gut Microbiota, Metabolites and Host Immunity. Nat. Rev. Immunol. 16, 341-352. doi:10.1038/nri.2016.42

Saitoh, O., and Nagayama, Y. (2006). Regulation of Graves' Hyperthyroidism with Naturally Occurring CD4+CD25+ Regulatory T Cells in a Mouse Model. Endocrinology 147, 2417-2422. doi:10.1210/en.2005-1024

Sato, K., Takahashi, N., Kato, T., Matsuda, Y., Yokoji, M., Yamada, M., et al. (2017). Aggravation of Collagen-Induced Arthritis by Orally Administered Porphyromonas Gingivalis through Modulation of the Gut Microbiota and Gut Immune System. Sci. Rep. 7, 6955. doi:10.1038/s41598-017-07196-7

Saulnier, D. M., Riehle, K., Mistretta, T. A., Diaz, M. A., Mandal, D., Raza, S., et al. (2011). Gastrointestinal Microbiome Signatures of Pediatric Patients with Irritable Bowel Syndrome. Gastroenterology 141, 1782-1791. doi:10.1053/j. gastro.2011.06.072

Schmidt, T. S. B., Raes, J., and Bork, P. (2018). The Human Gut Microbiome: From Association to Modulation. Cell 172, 1198-1215. doi:10.1016/j.cell.2018.02.044

Schwiertz, A., Taras, D., Schäfer, K., Beijer, S., Bos, N. A., Donus, C., et al. (2010). Microbiota and SCFA in Lean and Overweight Healthy Subjects. Obesity (Silver Spring) 18 (1), 190-195. doi:10.1038/oby.2009.167

Schwiertz, A., Taras, D., Schäfer, K., Beijer, S., Bos, N. A., Donus, C., et al. (2010). Microbiota and SCFA in Lean and Overweight Healthy Subjects. Obes. Silver Spring Md. 18, 190-195. doi:10.1038/oby.2009.167

Sekirov, I., Russell, S. L., Antunes, L. C., and Finlay, B. B. (2010). Gut Microbiota in Health and Disease. Physiol. Rev. 90 (3), 859-904. doi:10.1152/physrev.00045. 2009 
Shakya, M., Lo, C.-C., and Chain, P. S. G. (2019). Advances and Challenges in Metatranscriptomic Analysis. Front. Genet. 10, 904. doi:10.3389/fgene.2019. 00904

Sharon, G., Cruz, N. J., Kang, D.-W., Gandal, M. J., Wang, B., Kim, Y.-M., et al. (2019). Human Gut Microbiota from Autism Spectrum Disorder Promote Behavioral Symptoms in Mice. Cell 177, 1600-1618. e17. doi:10.1016/j.cell. 2019.05.004

Shi, N., Li, N., Duan, X., and Niu, H. (2017). Interaction between the Gut Microbiome and Mucosal Immune System. Mil. Med Res 4, 14. doi:10.1186/ s40779-017-0122-9

Shi, T.-T., Hua, L., Wang, H., and Xin, Z. (2019). The Potential Link between Gut Microbiota and Serum TRAb in Chinese Patients with Severe and Active Graves' Orbitopathy. Int. J. Endocrinol. 2019, 1-12. doi:10.1155/2019/9736968

Shi, T.-T., Xin, Z., Hua, L., Wang, H., Zhao, R.-X., Yang, Y.-L., et al. (2021). Comparative Assessment of Gut Microbial Composition and Function in Patients with Graves' Disease and Graves' Orbitopathy. J. Endocrinol. Invest. 44, 297-310. doi:10.1007/s40618-020-01298-2

Shi, T.-T., Xin, Z., Hua, L., Zhao, R.-X., Yang, Y.-L., Wang, H., et al. (2019). Alterations in the Intestinal Microbiota of Patients with Severe and Active Graves' Orbitopathy: a Cross-Sectional Study. J. Endocrinol. Invest. 42, 967-978. doi:10.1007/s40618-019-1010-9

Sircana, A., De Michieli, F., Parente, R., Framarin, L., Leone, N., Berrutti, M., et al. (2019). Gut Microbiota, Hypertension and Chronic Kidney Disease: Recent Advances. Pharmacol. Res. 144, 390-408. doi:10.1016/j.phrs.2018.01.013

Sivaprakasam, S., Prasad, P. D., and Singh, N. (2016). Benefits of Short-Chain Fatty Acids and Their Receptors in Inflammation and Carcinogenesis. Pharmacol. Ther. 164, 144-151. doi:10.1016/j.pharmthera.2016.04.007

Smith, T. J., and Hegedüs, L. (2016). Graves' Disease. N. Engl. J. Med. 375, 1552-1565. doi:10.1056/NEJMra1510030

Sommer, F., Anderson, J. M., Bharti, R., Raes, J., and Rosenstiel, P. (2017). The Resilience of the Intestinal Microbiota Influences Health and Disease. Nat. Rev. Microbiol. 15, 630-638. doi:10.1038/nrmicro.2017.58

Song, J., Shan, Z., Mao, J., and Teng, W. (2019). Serum Polyamine Metabolic Profile in Autoimmune Thyroid Disease Patients. Clin. Endocrinol. 90, 727-736. doi:10.1111/cen.13946

Struja, T., Eckart, A., Kutz, A., Huber, A., Neyer, P., Kraenzlin, M., et al. (2018). Metabolomics for Prediction of Relapse in Graves' Disease: Observational Pilot Study. Front. Endocrinol. 9, 623. doi:10.3389/fendo.2018.00623

Su, X., Yin, X., Liu, Y., Yan, X., Zhang, S., Wang, X., et al. (2020). Gut Dysbiosis Contributes to the Imbalance of Treg and Th17 Cells in Graves' Disease Patients by Propionic Acid. J. Clin. Endocrinol. Metab. 105, dgaa511. doi:10.1210/ clinem/dgaa511

Takeuchi, Y., Hirota, K., and Sakaguchi, S. (2020). Impaired T Cell Receptor Signaling and Development of $\mathrm{T}$ Cell-Mediated Autoimmune Arthritis. Immunol. Rev. 294, 164-176. doi:10.1111/imr.12841

Tan, J., McKenzie, C., Potamitis, M., Thorburn, A. N., Mackay, C. R., and Macia, L. (2014). The Role of Short-Chain Fatty Acids in Health and Disease. Adv. Immunol. 121, 91-119. doi:10.1016/B978-0-12-800100-4.00003-9

Teng, F., Felix, K. M., Bradley, C. P., Naskar, D., Ma, H., Raslan, W. A., et al. (2017). The Impact of Age and Gut Microbiota on Th17 and Thh Cells in K/BxN Autoimmune Arthritis. Arthritis Res. Ther. 19, 188. doi:10.1186/s13075-017-1398-6

Turnbaugh, P. J., and Gordon, J. I. (2009). The Core Gut Microbiome, Energy Balance and Obesity. J. Physiol. 587, 4153-4158. doi:10.1113/jphysiol.2009. 174136

Van Treuren, W., and Dodd, D. (2020). Microbial Contribution to the Human Metabolome: Implications for Health and Disease. Annu. Rev. Pathol. Mech. Dis. 15, 345-369. doi:10.1146/annurev-pathol-020117-043559

Vatanen, T., Kostic, A. D., d'Hennezel, E., Siljander, H., Franzosa, E. A., Yassour, M., et al. (2016). Variation in Microbiome LPS Immunogenicity Contributes to Autoimmunity in Humans. Cell 165, 842-853. doi:10.1016/j.cell.2016.04.007

Venkataraman, A., Sieber, J. R., Schmidt, A. W., Waldron, C., Theis, K. R., and Schmidt, T. M. (2016). Variable Responses of Human Microbiomes to Dietary Supplementation with Resistant Starch. Microbiome 4 (1), 33. doi:10.1186/ s40168-016-0178-x
Virili, C., Fallahi, P., Antonelli, A., Benvenga, S., and Centanni, M. (2018). Gut Microbiota and Hashimoto's Thyroiditis. Rev. Endocr. Metab. Disord. 19, 293-300. doi:10.1007/s11154-018-9467-y

Wang, W.-L., Xu, S.-Y., Ren, Z.-G., Tao, L., Jiang, J.-W., and Zheng, S.-S. (2015). Application of Metagenomics in the Human Gut Microbiome. Wjg 21, 803-814. doi:10.3748/wjg.v21.i3.803

Wang, Z., Zhang, Q., Lu, J., Jiang, F., Zhang, H., Gao, L., et al. (2010). Identification of Outer Membrane Porin F Protein ofYersinia enterocoliticaRecognized by Antithyrotopin Receptor Antibodies in Graves' Disease and Determination of its Epitope Using Mass Spectrometry and Bioinformatics Tools. J. Clin. Endocrinol. Metab. 95, 4012-4020. doi:10.1210/jc.2009-2184

Weiss, M., Ingbar, S. H., Winblad, S., and Kasper, D. L. (1983). Demonstration of a Saturable Binding Site for Thyrotropin in Yersinia Enterocolitica. Science 219, 1331-1333. doi:10.1126/science.6298936

Yan, H.-x., An, W.-c., Chen, F., An, B., Pan, Y., Jin, J., et al. (2020). Intestinal Microbiota Changes in Graves' Disease: a Prospective Clinical Study. Biosci. Rep. 40, BSR20191242. doi:10.1042/BSR20191242

Yang, M., Sun, B., Li, J., Yang, B., Xu, J., Zhou, X., et al. (2019). Alteration of the Intestinal flora May Participate in the Development of Graves' Disease: a Study Conducted Among the Han Population in Southwest China. Endocr. Connect. 8, 822-828. doi:10.1530/EC-19-0001

Yasuda, K., Kitagawa, Y., Kawakami, R., Isaka, Y., Watanabe, H., Kondoh, G., et al. (2019). Satb1 Regulates the Effector Program of Encephalitogenic Tissue Th17 Cells in Chronic Inflammation. Nat. Commun. 10, 549. doi:10.1038/s41467019-08404-w

Yuan, Q., Zhao, Y., Zhu, X., and Liu, X. (2017). Low Regulatory T Cell and High IL17 mRNA Expression in a Mouse Graves' Disease Model. J. Endocrinol. Invest. 40, 397-407. doi:10.1007/s40618-016-0575-9

Zhao, F., Feng, J., Li, J., Zhao, L., Liu, Y., Chen, H., et al. (2018). Alterations of the Gut Microbiota in Hashimoto's Thyroiditis Patients. Thyroid 28, 175-186. doi:10.1089/thy.2017.0395

Zheng, X., Xie, G., Zhao, A., Zhao, L., Yao, C., Chiu, N. H. L., et al. (2011). The Footprints of Gut Microbial-Mammalian Co-metabolism. J. Proteome Res. 10, 5512-5522. doi:10.1021/pr2007945

Zhou, L., Li, X., Ahmed, A., Wu, D., Liu, L., Qiu, J., et al. (2014). Gut Microbe Analysis between Hyperthyroid and Healthy Individuals. Curr. Microbiol. 69, 675-680. doi:10.1007/s00284-014-0640-6

Zhou, L., Zhang, M., Wang, Y., Dorfman, R. G., Liu, H., Yu, T., et al. (2018). Faecalibacterium Prausnitzii Produces Butyrate to Maintain Th17/Treg Balance and to Ameliorate Colorectal Colitis by Inhibiting Histone Deacetylase 1. Inflamm. Bowel Dis. 24, 1926-1940. doi:10.1093/ibd/ izy182

Zhu, Q., Hou, Q., Huang, S., Ou, Q., Huo, D., Vázquez-Baeza, Y., et al. (2021). Compositional and Genetic Alterations in Graves' Disease Gut Microbiome Reveal Specific Diagnostic Biomarkers. ISME J. 15, 3399-3411. doi:10.1038/ s41396-021-01016-7

Conflict of Interest: The authors declare that the research was conducted in the absence of any commercial or financial relationships that could be construed as a potential conflict of interest.

Publisher's Note: All claims expressed in this article are solely those of the authors and do not necessarily represent those of their affiliated organizations, or those of the publisher, the editors, and the reviewers. Any product that may be evaluated in this article, or claim that may be made by its manufacturer, is not guaranteed or endorsed by the publisher.

Copyright $\odot 2022 \mathrm{Liu}, \mathrm{Liu}, \mathrm{Liu}$, Shang, Wei and Yin. This is an open-access article distributed under the terms of the Creative Commons Attribution License (CC BY). The use, distribution or reproduction in other forums is permitted, provided the original author(s) and the copyright owner(s) are credited and that the original publication in this journal is cited, in accordance with accepted academic practice. No use, distribution or reproduction is permitted which does not comply with these terms. 